\title{
Facilitadores en el deporte paralímpico: motivos de práctica deportiva en jugadores con discapacidad física y visual \\ Facilitators in Paralympic sport: motives for sports practice in players with physical and visual disabilities
}

\author{
Maria Alejandra Zambrano Palencia, Olga Lucia Hincapié Gallón \\ Institución Universitaria Escuela Nacional del Deporte (Colombia)
}

\begin{abstract}
Resumen. La motivación como un elemento propio de cada deportista es generadora de respuestas positivas en la práctica deportiva, por tanto, la evaluación de esta en los deportistas adaptados contribuye a generar propuestas que consideren las expectativas de los mismos. Objetivo: Identificar los motivos de la práctica deportiva en jugadores de tenis en silla de ruedas y goalball. Método: Quince deportistas: 5 de tenis en silla de ruedas y 10 de goalball, pertenecientes a diferentes clubes regionales, respondieron el Cuestionario «Participation Motivation Inventory». Resultados: El 100\% de los jugadores en tenis en silla de ruedas valoraron muy importante los ítems: «Me gusta ganar», «Quiero estar en forma», «Me gusta hacer ejercicio», «Me gusta la acción», «Me gusta la competición», «Me gusta practicar deporte», «Quiero estar físicamente bien», $\mathrm{y}$ «Quiero alcanzar un status - elite»; mientras que en goalball fueron los ítems: «Quiero mejorar mis habilidades», «Quiero aprender nuevas habilidades», «Me gusta la competición», «Me gusta practicar deporte», «Quiero mejorar mi nivel», «Quiero estar físicamente bien»y «Me gusta divertirme». En ambas poblaciones los ítems: «Es por satisfacer a mis padres o amigos» $\mathrm{y}$ «Por influencia de los entrenadores o instructores» fueron valorados como nada importante por el 40\% de los deportistas. Conclusiones: Los motivos más valorados por los jugadores de tenis en silla de ruedas y los jugadores de goalball están relacionados con el factor de superación y el factor personal respectivamente, mientras que el factor socio-deportivo es el menos importante en su motivación para la práctica deportiva.
\end{abstract}

Palabras claves: motivación, deporte, discapacidad, tenis en silla de ruedas, goalball.

Abstract. Motivation as an element of each athlete generates positive responses in practice, therefore, its evaluation in adapted athletes contributes to generating proposals that consider their sporting expectations. Aims: To identify the motives for sports practice in wheelchair tennis and goalball players. Method: Fifteen athletes: 5 for wheelchair tennis and 10 for goalball, belonging to different clubs in the region, answered the Questionnaire «Participation Motivation Inventory». Results: $100 \%$ of wheelchair tennis players rated the items as very important: «I like to win», «I want to be fit», «I like to exercise», «I like action», «I like the competition «,» I like to practice sports «,» I want to be physically well «, and» I want to achieve elite status «; while in goalball there were the items: «I want to improve my skills», «I want to learn new skills», «I like competition», «I like to practice sports», «I want to improve my level», «I want to be physically well» and «I like to have fun». In both populations the items: «It is to satisfy my parents or friends» and «Due to the influence of the coaches or instructors» were valued as not important by $40 \%$ of the athletes. Conclusions: The motives most valued by wheelchair tennis players and goalball players are related to the overcoming factor and the personal factor respectively, while the socio-sports factor is the least important in their motivation for sports practice.

Keywords: motivation, sport, disability, wheelchair tennis, goalball.

\section{Introducción}

La motivación ha sido descrita como un proceso activo, intencional y hacia una meta, donde interactúan factores personales y ambientales, que afectan la selección, intensidad y persistencia de un individuo y que, en el caso del deporte, interfiere directamente con la calidad del rendimiento del atleta (Freire da Silva, Almeida

Fecha recepción: 03-07-21. Fecha de aceptación: 05-10-21

Maria Alejandra Zambrano Palencia

maria.zambrano@endeporte.edu.co
Da Silva, de Souza Duim, Andrade do Nascimento, \& Ferreira de Araújo, 2019).

La motivación como factor propio de cada persona se convierte en un componente promotor de los procesos de respuesta a los diferentes estímulos para la práctica deportiva. Solo desde comprender la diferencia existente en lo referente a que motiva a una u otra persona se pueden direccionar procesos rescatando las expectativa propias del deportista, y que para el deportista adaptado es igual, y más cuando desde la misma discapacidad se pueden direccionar los motivos para la práctica (Muñoz, Garrote, \& Sánchez, 2017) lo que con- 
lleva a realizar deporte convirtiendo el reconocimiento de los motivos en un factor diferenciador en el momento de hacer las propuestas de actividades a los deportistas.

La práctica deportiva en las personas con discapacidad, es una herramienta importante que contribuye al desarrollo social de las personas y a la adquisición estilos de vida saludables (K1zar, Demir, \& Genç, 2021), y específicamente en deportistas de nivel competitivo la permanencia en el deporte genera sentido y significado en sus vidas (Vieira, Colere, \& Souza, 2021). Adicionalmente, el deporte ha sido un catalizador para la reestructuración de las identidades, empoderamiento y resistencia hacia actitudes discapacitantes, dando una visión más coherente en la comprensión de la discapacidad (Hernández et al., 2021). Aunque se ha identificado que tanto las personas con y sin discapacidad practican deporte con fines de disfrute, beneficios en la salud y los aspectos sociales asociados (McLoughlin, Weisman Fecske, Castaneda, Gwin, \& Graber, 2017), los primeros presentan una baja participación en programas relacionado con la actividad física, el deporte o la recreación (Jimenez \& Cárdenas, 2020), por lo cual es importante fortalecer las acciones de los profesionales en salud, especialmente del fisioterapeuta, dado que el periodo de rehabilitación es reconocido como el espacio donde las personas con discapacidad física se acercan al deporte (Hernández et al., 2021).

En la evolución de la práctica deportiva adaptada se han identificado avances para América Latina lo que se ve reflejado en el aumento de las medallas logradas, esto de la mano del aumento de la población con discapacidad, que para América latina y el caribe se reporta está en un 12,6\% (CEPAL, 2014) y para Colombia en un 7,1\% (DANE, 2018), lo que invita cada día a considerar cada uno de los aspectos que pueden jugar algún tipo de impacto en el desempeño; y más allá la posibilidad de la práctica deportiva adaptada con el reconocimiento de la multidimensionalidad del ser humano en la enseñanza de la fisioterapia deportiva, haciéndose necesario revisar a profundidad el tema de motivación en esta población y más cuando el trabajo que inspiró la investigación es en la población con discapacidad de un sector de Colombia que practica deporte.

La motivación en el ámbito deportivo vista como un factor personal, tiene una incidencia dentro del actuar del deportista pues se relaciona con aspectos de participación, desempeño y permanencia en el deporte. Dado que el modelo biopsicosocial junto con la CIF soporta la práctica deportiva en personas con discapacidad y aunado a la evolución del quehacer profesional del fisioterapeuta en esta población (Silva, Vital, \& Túlio de Mello, 2016), se hace inminente reconocer factores como la motivación mirada desde sus posibilidades, esto respetando que la profundidad en el tema se encuentra en cabeza de psicología con objetivos propios más relacionados con el comportamiento, igualmente para otros profesionales del equipo multidisciplinar como el fisioterapeuta tener presente estos factores es un complemento que permite abordajes acordes a las necesidades del deportista. Por tanto, se planteó como objetivo general del proyecto de pasantía del programa de fisioterapia de la Institución Universitaria Escuela Nacional del deporte, el identificar los motivos de la práctica deportiva en jugadores de tenis en silla de ruedas y goalball de diferentes clubes de la región; los hallazgos de esta investigación son divulgados como una experiencia significativa resultado del proyecto mencionado con anterioridad,

\section{Método}

Estudio con enfoque cuantitativo, de tipo descriptivo y corte trasversal, cuyo objetivo fue identificar los motivos de la práctica deportiva en jugadores de tenis en silla de ruedas y goalball a través del diligenciamiento del cuestionario «Participation Motivation Inventory». Para esta investigación se seleccionó una muestra por conveniencia donde se incluyeron 15 deportistas: 5 jugadores de tenis en silla de ruedas ( 5 hombres con edades entre los 14 y 49 años) y 10 jugadores de goalball (5 hombres y 5 mujeres con edades entre los 20 y 52 años), con mínimo 2 meses de entrenamiento, dos veces por semana y pertenecientes a diferentes clubes del Valle del Cauca, se excluyeron deportistas que practicaran otra modalidad adicional al tenis en silla de ruedas o goalball.

El planteamiento y desarrollo de este estudio fue resultado de un proyecto de pasantía del programa de pregrado de fisioterapia de la Institución Universitaria Escuela Nacional del deporte en el 2018, y contribuyo para el macroproyecto «Análisis comparativo cinemático y funcional de cualidades físicas entre deportistas convencionales y con lesión medular a nivel competitivo»; con aprobación del comité de ética de la institución con memorando No 4,1.17.03.119. Durante el desarrollo de este estudio se reconoce que los deportistas cuentan con el acompañamiento del psicólogo como parte del equipo interdisciplinar. 


\section{Instrumento}

Se utilizó el Cuestionario «Participation Motivation Inventory», el cual ha sido aplicado previamente en deportistas con y sin discapacidad (Durand, 1988; Balaguer \& Atienza, 1994; Lázaro, Villamarín, \& Limonero, 1996; Cecchini, Méndez, \& Muñiz, 2002; Torralba, Braz, \& Rubio, 2014; Torralba, Vieira, \& Rubio, 2017) usando la última versión adaptado al deporte Paralímpico en español, con una fiabilidad total del cuestionario igual a 0.852 y para los cuatro factores un á de Cronbach entre 0.7 y 0.8 , lo cual indica una consistencia interna valida (Torralba et al., 2017). El cuestionario está integrado por 30 ítems valorados por una escala tipo Likert, en el cual se les pregunta a los participantes que tan importante es para ellos cada uno de los motivos para practicar deporte, indicando si creen que ese motivo es «muy importante» (1), «algo importante» (2) o «nada importante» (3).

\section{Recolección de los datos}

El cuestionario fue autodiligenciado por los 5 jugadores de tenis en silla de ruedas al finalizar una de sus sesiones de entrenamiento. Previo al diligenciamiento, el investigador explico por separado a cada uno de los deportistas los objetivos del estudio, facilitando las orientaciones de la ejecución y relleno del cuestionario. Los deportistas diligenciaron el cuestionario en espacios separados y el investigador estuvo disponible para resolver cualquier duda que pudiera plantearse.

Los 10 jugadores de goalball también diligenciaron el cuestionario al finalizar una de sus sesiones de entrenamiento con ayuda de un auxiliar quien leía los ítems a cada jugador por separado, y registraba el nivel de importancia (1, 2 o 3) según su respuesta. Fueron 5 auxiliares quienes se entrenaron previamente de forma que éstos supiesen transmitir correctamente cada ítem al encuestado y registrar adecuadamente sus respuestas. En todo momento se señaló la voluntariedad, la finalidad y el anonimato de los resultados de la investigación, y así mismo el investigador estuvo pendiente ante cualquier inquietud.

\section{Análisis estadístico}

Se procedido a la valoración del cuestionario a partir de la última estructura utilizada por Torralba et al. (2017), quienes tras un análisis factorial de los ítems con método de componentes principales y rotación varimax obtuvieron una estructura factorial de 4 componentes interpretables distribuidos de la siguiente forma: Inte- gración social con 9 ítems, Personal con 6 ítems, Sociodeportivo con 8 ítems, y Superación con 7 ítems. Para el análisis de la información se utilizó el paquete estadístico IBM $®$ SPSS Statistics V20, en el cual se aplicó estadística descriptiva con frecuencias, medias y desviación estándar a los 30 ítems que componen el Cuestionario «Participation Motivation Inventory», y los 4 factores motivacionales mencionados anteriormente.

\section{Resultados}

Tenis en silla de ruedas: De acuerdo a la tabla 1, se presentan las respuestas de los 30 ítems agrupados en los 4 factores (personal, integración social, superación, socio deportivo), evaluados según la media, frecuencia y porcentaje de acuerdo al tipo de importancia ( $1=$ muy importante, 2= Algo importante, 3= Nada importante). El 100\% de los deportistas $(n=5)$ valoraron como «muy importante» los ítems: «Me gusta ganar», «Quiero estar en forma», «Me gusta hacer ejercicio», «Me gusta la acción», «Me gusta la competición», «Me gusta practicar deporte», «Quiero estar físicamente bien», y «Quiero alcanzar un status - elite»; mientras que los ítems «Me gustan los desafíos» $\mathrm{y}$ «Es por satisfacer a mis padres o amigos» fueron considerados como «nada importante» por el $40 \%$ de los deportistas.

Según lo muestra la tabla 2, el factor motivacional con mayor puntuación en los deportistas de tenis en

\begin{tabular}{|c|c|c|c|c|c|c|c|}
\hline \multirow[t]{2}{*}{ Factores } & \multirow[t]{2}{*}{ Media } & \multicolumn{2}{|c|}{$\begin{array}{c}\text { Muy } \\
\text { importante }\end{array}$} & \multicolumn{2}{|c|}{$\begin{array}{c}\text { Algo } \\
\text { importante }\end{array}$} & \multicolumn{2}{|c|}{$\begin{array}{c}\text { Nada } \\
\text { importante }\end{array}$} \\
\hline & & $\mathrm{F}$ & $\%$ & $\mathrm{~F}$ & $\%$ & $\mathrm{~F}$ & $\%$ \\
\hline \multicolumn{8}{|l|}{ Integración social } \\
\hline 2. Quiero estar con mis amigos & 1,60 & 2 & 40 & 3 & 60 & - & - \\
\hline 4. Quiero gastar energía & 1,20 & 4 & 80 & 1 & 20 & & \\
\hline 8. Me gusta el trabajo en equipo & 1,60 & 3 & 60 & 1 & 20 & 1 & 20 \\
\hline 11. Me gusta hacer nuevos amigos & 1,80 & 1 & 20 & 4 & 80 & & \\
\hline 13. Quiero relajarme & 1,80 & 2 & 40 & 2 & 40 & 1 & 20 \\
\hline 16. Me gusta tener algo que hacer & 1,20 & 4 & 80 & 1 & 20 & - & - \\
\hline 18. Me gusta el espíritu de equipo & 1,60 & 3 & 60 & 1 & 20 & 1 & 20 \\
\hline 19. Me gusta salir de casa & 1,60 & 3 & 60 & 1 & 20 & 1 & 20 \\
\hline 30. Me gusta utilizar el equipo o instalaciones & 1,40 & 4 & 80 & 1 & 20 & - & - \\
\hline \multicolumn{8}{|l|}{ Personal } \\
\hline 1. Quiero mejorar mis habilidades & 1,40 & 4 & 80 & - & - & 1 & 20 \\
\hline 6. Quiero estar en forma & 1,00 & 5 & 100 & - & - & & - \\
\hline 10. Quiero aprender nuevas habilidades & 1,60 & 3 & 60 & 1 & 20 & 1 & 20 \\
\hline 23. Quiero mejorar mi nivel & 1,40 & 4 & 80 & 1 & 20 & - & - \\
\hline 24. Quiero estar físicamente bien & 1,00 & 5 & 100 & - & & & - \\
\hline 29. Me gusta divertirme & 1,20 & 4 & 80 & 1 & 20 & - & - \\
\hline \multicolumn{8}{|l|}{ Superación } \\
\hline 3. Me gusta ganar & 1,00 & 5 & 100 & - & - & - & - \\
\hline 7. Me gustan las emociones & 1,40 & 3 & 60 & 2 & 40 & & \\
\hline 15. Me gusta hacer ejercicio & 1,00 & 5 & 100 & - & - & & - \\
\hline 17. Me gusta la acción & 1,00 & 5 & 100 & & & & \\
\hline 20. Me gusta la competición & 1,00 & 5 & 100 & & & & \\
\hline 22. Me gusta practicar deporte & 1,00 & 5 & 100 & - & - & - & - \\
\hline 26. Me gustan los desafíos & 1,80 & 3 & 60 & - & - & 2 & 40 \\
\hline \multicolumn{8}{|l|}{ Socio-deportivo } \\
\hline 5. Me gusta viajar & 1,20 & 4 & 80 & 1 & 20 & - & - \\
\hline 9. Es por satisfacer a mis padres & 2,00 & 2 & 40 & 1 & 20 & 2 & 40 \\
\hline 12. Me gusta hacer algo en lo que destaco & 1,20 & 4 & 80 & 1 & 20 & - & \\
\hline 14. Me gustan las recompensas & 1,60 & 3 & 60 & 1 & 20 & 1 & 20 \\
\hline 21. Me gusta sentirme importante & 1,60 & 3 & 60 & 1 & 20 & 1 & 20 \\
\hline 25. Quiero ser popular & 1,80 & 2 & 40 & 2 & 40 & 1 & 20 \\
\hline 27. Por influencia de los entrenadores & 1,80 & 2 & 40 & 2 & 40 & 1 & 20 \\
\hline 28. Quiero alcanzar un status-élite & 1,00 & 5 & 100 & - & - & - & - \\
\hline
\end{tabular}


Tabla 2.

Análisis descriptivo factores motivacionales de los jugadores de tenis en silla de ruedas

\begin{tabular}{ccc} 
Análisis descriptivo factores motivacionales de los jugadores de tenis en silla de ruedas & \\
\hline Factores & Media & Desv. Típ. \\
\hline Superación & 1,17 & 0,19 \\
Personal & 1,27 & 0,42 \\
Integración social & 1,50 & 0,39 \\
Socio deportivo & 1,53 & 0,47
\end{tabular}

silla de ruedas es el factor superación, seguido del personal e integración social y por último el factor socio-deportivo con la valoración más baja.

Goalball: Según lo expuesto en la tabla 3, se encuentra que todos los deportistas $(\mathrm{n}=10)$ consideran «muy importante» los ítems: «Quiero mejorar mis habilidades», «Quiero aprender nuevas habilidades»,»Me gusta la competición», «Me gusta practicar deporte», «Quiero mejorar mi nivel», «Quiero estar físicamente bien», «Me gusta divertirme»; mientras que los ítems «Por influencia de los entrenadores o instructores»y «Es por satisfacer a mis padres o amigos» fueron valorados como «nada importante» por el 40\% y 50\% de los deportistas con discapacidad visual respectivamente.

Por otro lado, como lo muestra la tabla 4, el factor con mayor valoración es el personal, siguiéndole superación, integración social y por último el factor socio-deportiVO.

Tabla 3.

Análisis descriptivo de las respuestas por item agrupado según factores en los jugadores de goalball

\begin{tabular}{lccccccc}
\hline \multirow{2}{*}{ Factores } & \multicolumn{6}{c}{ Muy } & \multicolumn{2}{c}{ Algo } & \multicolumn{2}{c}{ Nada } \\
& Media & importante & importante & importante \\
\cline { 2 - 7 } & & F & $\%$ & F & $\%$ & F & $\%$ \\
\hline Integración social & & & & & & \\
\hline 2. Quiero estar con mis amigos & 1,10 & 9 & 90 & 1 & 10 & - & -
\end{tabular}

4. Quiero estar con mis amigos

8. Me gusta el trabajo en equip

11. Me gusta hacer nuevos amigos

13. Quiero relajarme

16. Me gusta tener algo que hacer

18. Me gusta el espíritu de equipo

19. Me gusta salir de casa

$\begin{array}{lllll}1,10 & 9 & 90 & 1 & 10 \\ 1,30 & 7 & 70 & 3 & 30\end{array}$

$\begin{array}{lllllll}1,50 & 7 & 70 & 1 & 10 & 2 & 20\end{array}$

$\begin{array}{lllll}1,10 & 9 & 90 & 1 & 10\end{array}$

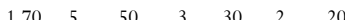

30. Me gusta utilizar el equipo o instalaciones

Personal

1. Quiero mejorar mis habilidades

6. Quiero estar en forma

10. Quiero aprender nuevas habilidades

23. Quiero mejorar mi nivel

24. Quiero estar físicamente bien

29. Me gusta divertirme

Superación

3. Me gusta gana

$\begin{array}{llllll}\text { 7. Me gustan lo } & 1,10 & 9 & 90 & 1 & 10 \\ & 1,10 & 9 & 90 & 1 & 10\end{array}$

15. Me gusta hacer ejercicio

17. Me gusta la acción

20. Me gusta la competición

22. Me gusta practicar deporte

26. Me gustan los desafíos

$\begin{array}{lllll}, 30 & 7 & 70 & 3 & 30\end{array}$

$\begin{array}{lllllll}1,50 & 7 & 70 & 1 & 10 & 2 & 20\end{array}$

$\begin{array}{lllll}1,10 & 9 & 90 & 1 & 10\end{array}$

$\begin{array}{lllll}1,50 & 5 & 50 & 5 & 50\end{array}$

$\begin{array}{ccccc}1,00 & 10 & 100 & - & -\end{array}$

$1,10 \quad 9=90$

$\begin{array}{lll}1,00 & 10 & 100 \\ 1,00 & 10 & 100\end{array}$

$\begin{array}{lll}1,00 & 10 & 100\end{array}$

$\begin{array}{lll}1,00 & 10 \quad 100\end{array}$

Socio-deportivo

5. Me gusta viajar

9. Es por satisfacer a mis padres

12. Me gusta hacer algo en lo que destaco

14. Me gustan las recompensas

21. Me gusta sentirme importante

25. Quiero ser popular

27. Por influencia de los entrenadore

28. Quiero alcanzar un status-élite

$\begin{array}{lllll}1,10 & 9 & 90 & 1 & 10\end{array}$

$\begin{array}{lllll}1,40 & 6 & 60 & 4 & 40\end{array}$

$\begin{array}{lllll}1,20 & 8 & 80 & 2 & 20\end{array}$

$\begin{array}{lll}1,00 & 10 \quad 100\end{array}$

$\begin{array}{lll}1,00 & 10 & 100\end{array}$

$\begin{array}{lllll}1,10 & 9 & 90 & 1 & 10\end{array}$

$\begin{array}{lllll}1,10 & 9 & 90 & 1 & 10 \\ 2,30 & 2 & 20 & 3 & 30 \\ 1,10 & 9 & 90 & 1 & 10\end{array}$

$\begin{array}{lllll}2,30 & 2 & 20 & 3 & 30\end{array}$

$\begin{array}{lllll}1,10 & 9 & 90 & 1 & 10\end{array}$

$\begin{array}{lllll}1,30 & 7 & 70 & 3 & 30 \\ 1,60 & 6 & 60 & 2 & 20\end{array}$

$\begin{array}{lllllll}1,60 & 6 & 60 & 2 & 20 & 2 & 20\end{array}$

$\begin{array}{lllllll}1,80 & 5 & 50 & 2 & 20 & 3 & 30\end{array}$

$\begin{array}{lllllll}2,20 & 2 & 20 & 4 & 40 & 4 & 40\end{array}$

Tabla 4

Análisis descriptivo factores motivacionales de los jugadores de goalball

\begin{tabular}{ccc}
\hline Factores & Media & Desv. Típ. \\
\hline Personal & 1,02 & 0,05 \\
Superación & 1,13 & 0,16 \\
Integración social & 1,30 & 0,28 \\
Socio deportivo & 1,57 & 0,37 \\
\hline
\end{tabular}

\section{Discusión}

La motivación vista como un facilitador cumple el papel de directora de la conducta afectando la elección, participación, rendimiento y continuación de la participación deportiva de un individuo. Con el fin de identificar los motivos de la práctica deportiva en jugadores de tenis en silla de ruedas y goalball, y posterior al análisis de las respuestas obtenidas a través del cuestionario «Participation Motivation Inventory», adaptado al deporte Paralímpico en su versión en español, los hallazgos indican que la motivación principal de los deportistas de tenis en silla de ruedas gira alrededor del factor superación, es decir que su motivación está relacionada con el ego, la victoria, la acción, la competencia y ser activo físicamente, mientras que en los jugadores de goalball el factor personal obtuvo la mayor importancia, indicando que la motivación se orienta a demostrar un buen dominio de la tarea misma, mejorar las habilidades, así como aprender nuevos movimientos y mejorar el nivel deportivo. Por otro lado, se encuentra que para ambas poblaciones el factor menos motivante es el socio-deportivo, encontrándose que la estima de terceros como entrenadores, padres o amigos no es relevante en su motivación para la práctica deportiva.

Estos mismos resultados concuerdan con los reportes de investigaciones que han utilizado el mismo cuestionario en deportistas con discapacidad (Torralba et al., 2014, 2017), donde los ítems con mayor nivel de importancia demuestran un inclinación de los deportistas hacia los factores personal y superación; del mismo modo, ítems como: «Por influencia de los entrenadores o instructores» $\mathrm{y}$ «Es por satisfacer a mis padres o amigos» son valorados con poco importancia por los deportistas. Si bien esto indica una motivación a la práctica deportiva donde la estima de terceros pasa a un segundo plano, se ha documentado la importancia del entrenador en las primeras etapas de la vida del deportista (González, Sánchez, \& Márquez, 2000; Gould, Feltz, \&Weiss, 1985), así como su papel fundamental en la generación de un clima motivacional hacia la tarea en el que el deportista pueda ser más autónomo (López-Walle, Balaguer, Castillo, \& Tristán, 2011; Marcos, Sánchez, Sánchez, Amado, \& García, 2009), lo que puede explicar estos hallazgos dado que los deportistas de las muestras anteriores ya han pasado su periodo de iniciación deportiva.

Por otro lado, la motivación en el ámbito deportivo también ha sido descrita desde la motivación extrínseca e intrínseca; la primera determinada por recompensas o agentes externos que no están directamente relacio- 
nada con la tarea a realizar; y la segunda relacionada con la necesidad de explorar el entorno, la curiosidad y el placer que se experimenta al realizar una actividad sin recibir una gratificación externa directa. El estudio realizado en deportistas colombianos con discapacidad visual (Urrea \& Guillén, 2016) revelaron que el género masculino puntuaba más alto en regulación externa que el femenino, similar a otros estudios en deportistas sin discapacidad donde las mujeres puntúan una motivación intrínsecamente más alta demostrando un perfil más autodeterminado comparado con los hombres (Chantal, Guay, Dobreva-Martinova, \& Vallerand, 1996; Balaguer, Castillo, \& Duda, 2003; Pelletier et al., 1995). A pesar que el objetivo de esta investigación no planteaba encontrar diferencias en el género, todos los jugadores de tenis en silla de ruedas eran hombres y puntuaron con mayor importancia en el factor de superación el cual contempla ítems como: «Me gusta la competición» «Me gustan los desafíos» o «Me gusta ganar», que pueden ser asociados a la motivación extrínseca de tipo introyectada, en la cual el individuo ejerce presión sobre sí mismo para regular su comportamiento (Urrea \& Guillén, 2016). No obstante, de los escasos estudios de motivación en deportistas con discapacidad que han analizado posibles diferencias entre género, aun se presentan controversias (Torralba et al., 2014, 2017; Muñoz et al., 2017), posiblemente a la heterogeneidad de las muestras en cuanto a tipo de discapacidad, nacionalidad y nivel deportivo.

Así mismo, se destacan los diferentes enfoques del deporte en relación a su objetivo de alcance: deporte rehabilitador, terapéutico, recreativo, competición y de riesgo, tal como lo propone la pirámide del deporte adaptado de García de Mingo (1992). En etapas tempranas la motivación está relacionada con el propósito de mejorar la salud, el estado físico y la construcción de redes de apoyo (Jeong \& Park, 2013), como lo demuestran Muñoz et al. (2017) quienes han reportado que los motivos de práctica deportiva a nivel terapéutico o por ocio son querer estar en buena forma física por salud y autoimagen, conocer personas nuevas, estar con amigos, subir la autoestima y porque les gusta y disfrutan hacer esa actividad; similar a Gutiérrez-Sanmartín \& Caus-Pertegáz (2006) donde valoraron más la posibilidad de crear nuevas amistades, viajar, sentirse necesarios y ayudar a los demás. Pese a que la población del presente estudio practica deporte a nivel competitivo, y más del 90\% ha valorado como muy importante el ítem: «Quiero alcanzar un status-élite»; los jugadores de goalball llevan menos tiempo practicando deporte y han competido solo a nivel nacional, por tanto, pudieron calificar con mayor importancia aquellos aspectos relacionados a tener una buena condición física, mejorar su nivel y habilidades, en contraste con los jugadores de tenis en silla de ruedas quienes tienen más años de experiencia deportiva y la mayoría ha competido a nivel internacional; no obstante la evidencia actual, deja vislumbrar diferencias en los motivos de la práctica deportiva dependiendo del nivel u enfoque, pero no por los años de práctica (Torralba et al., 2017; Urrea \& Guillén, 2016), lo cual brindan una perspectiva a los profesionales inmersos en el área del deporte adaptado para direccionar la práctica en sus diferentes niveles (terapéutico, recreativo o competitivo) según los motivos y aspiraciones de los deportistas.

En relación a la modalidad del deporte (individualcolectivo), tenistas koreanos en silla de ruedas (Jeong \& Park, 2013) informaron una menor motivación hacia el disfrute de la actividad comparado con jugadores no coreanos, mientras que atletas brasileros de baloncesto, rugby y balonmano en silla de ruedas (Freire da Silva et al., 2019) indicaron una mayor orientación motivacional hacia la tarea, lo que va en línea con los reportes de este estudio. Así mismo en deportistas paralímpicos españoles (Torralba et al., 2017) han hallado diferencias significativas a favor de los deportes colectivos en los ítems «Quiero estar con mis amigos», «Quiero gastar energía», «Me gusta el trabajo en equipo»y «Me gusta el espíritu de equipo» y a favor de los deportes individuales en los ítems «Me gustan las recompensas», siendo estos mismos ítems igualmente valorados como muy importantes por el 70-90\% los jugadores de goalball de la presente investigación.

Aunque existen reportes en jugadores sin discapacidad (Moreno, Cervello, \& Gonzales, 2007), donde los jugadores de deportes colectivos tienen una mayor orientación al ego, y los deportes individuales una mayor orientación hacia la tarea, no parece existir la misma tendencia en los deportistas con discapacidad, quienes también puntúan más alto en lo referido al factor de integración social (Brasile, Kleiber, \& Harnisch, 1991; White \& Duda, 1993), junto con una mayor orientación al ego (Gutiérrez-Sanmartín \& Caus-Pertegáz, 2006) en comparación con deportistas sin discapacidad. Esto parece ser una parte fundamental para el proceso de inclusión ya que la construcción de una identidad deportiva contribuye a su reafirmación social, además de la incorporación de estilos de vida saludables (Muñoz et al., 2017; Úbeda-Colomer, Molina-Alventosa, \& Campos-Granell, 2016) 
Si bien existen diferentes teorías de la motivación, es importante destacar que la motivación es producto de un conjunto de variables sociales, ambientales e individuales que determinan o dirigen una conducta, y analizándola dentro del contexto de discapacidad y deporte adaptado, puede considerársele un elemento facilitador para la práctica deportiva (Úbeda-Colomer et al., 2016) y elemento clave para el compromiso y adherencia a la práctica deportiva (Iso-Ahola \& Clair, 2000), como expresan Carrasco et al. (2013) «es como una fuerza interna que nos provoca actuar, que nos mueve y nos mantiene en la acción», en donde convergen tanto el mismo deportista, el entrenador, las características del entrenamiento y los materiales».

\section{Conclusiones}

Las calificaciones otorgadas por los jugadores de tenis en silla de ruedas a los diferentes ítems dieron importancia al ganar, estar en forma, gusto por el ejercicio, la acción, la competición y el deporte y el querer alcanzar un status-elite, lo cual indica que los motivos más relevantes para este deporte estuvieron relacionados con el factor superación. En cuanto a los jugadores de goalball, estos consideraron muy importantes aspectos relacionados al mejorar y aprender nuevas habilidades, gusto por la competición, el deporte y la diversión, mejorar su nivel y estar físicamente bien, lo cual corresponde principalmente a una motivación guiada por el factor personal. Para ambos grupos el factor socio-deportivo fue el menos importante en la motivación para la práctica deportiva, por lo que la influencia de terceros paso a un segundo plano. A través del reconocimiento de un perfil motivacional que dirige la conducta de los deportistas es posible ampliar el panorama al momento de plantear propuestas que vayan encaminadas no solo a la mejora de su desempeño según el nivel, sino también acorde con la visión del individuo en relación con su propia práctica deportiva, incentivando preferiblemente una motivación enfocada más hacia la actividad misma que a las recompensas.

Esta investigación es una invitación para que los profesionales en ciencias de la salud y del deporte que realizan abordajes en el deportista paralímpico, identifiquen cuales son esos motivos que incentivan la práctica del deporte para así complementar y direccionar las intervenciones teniendo en cuenta la visión del deportista. Por otro lado, se recomienda para futuras investigaciones utilizar muestras más numerosas para que los resultados puedan ser más representativos, igualmente sería interesante la inclusión de otros deportes individuales y colectivos, practicados por población con discapacidad física, visual, auditiva e intelectual, y así poder establecer posibles diferencias o similitudes entre estos.

\section{Declaraciones}

Conflicto de intereses: Los autores declaran no existe conflicto de interés.

\section{Referencias}

Balaguer, I., \& Atienza, F. (1994). Principales motivos de losjóvenes para jugar al tenis. Apunts Sports Medicine, 31(122), 285300. Retrieved from https://www.apunts.org/en-pdfX0213371794055013

Balaguer,I., Castillo, I., \& Duda, J.L. (2003).LaEscala de Motivación Deportiva: unanueva medida de la motivaciónintrínseca, motivación extrínsecayno-motivación. Análisispsicométrico de la versión española. Proceedings of the II Congreso Internacional de Psicología Aplicada Al Deporte, 165.

Brasile, F. M., Kleiber, D. A., \& Harnisch, D. (1991). Analysis of participation incentives among athletes with and without disabilities. Therapeutic Recreation Journal, 25(1), 18-33. Retrieved from https: / /www.cabdirect.org/cabdirect/ abstract/19911887442

Carrasco, D., Carrasco, D. \& \& Carrasco, D. (2013). Psicología del deporte. Universidad Politécnica de Madrid. Instituto Nacional de Educación Física. Retrieved from http:// futbolcarrasco.com/wp-content/uploads/2014/08/ futbolcarrascoinef4curso4.pdf

Cecchini, J. A., Méndez, A., \& Muñiz, J. (2002). Motives for practicingsportin Spanish schoolchildren. Psicothema, 14(3). Retrieved from http://www.psicothema.com/pdf/760.pdf CEPAL. (2014). Informe regional sobre la medición de la discapacidad. Una mirada a los procedimientos de medición de la discapacidad en América Latina y el Caribe. Grupo de tareas sobre medición de la discapacidad Conferencia Estadística de lasAméricas(CEA). CEPAL. Retrieved from https://repositorio.cepal.org//handle/ 11362/36906

Chantal,Y., Guay,F.,Dobreva-Martinova,T., \&Vallerand,R.J.(1996). Motivationand eliteperformance/: an exploratoryinvestigation with Bulgarian athletes. International Journal of Sport Psychology, 27, 173-182. Retrieved from https://corpus.ulaval.ca/ jspui/handle/20.500.11794/13983

DANE. (2018). FUNCIONAMIENTO HUMANO: RESULTADOS CENSO NACIONAL DE POBLACIÓNYVIVIENDA 2018. Bogotá. Colombia.

Durand, M. (1988). Motivación y práctica deportiva en el niño. Madrid: Ed. Paidós.

FreiredaSilva,F.,AlmeidaDaSilva,J.,deSouzaDuim, G.A.,Andrade 
doNascimento,J.R., \& Ferreira deAraújo,P.(2019). Orientação motivacional de atletas usuários de cadeira de rodas. Caderno de Educação Física e Esporte, 17(2), 13-19. doi: 10.36453/23185104.2019.v17.n2.p13

Garcia de Mingo, J. A. (1992). Actividades fisicas y deporte para minusválidos. Campomanes Libros.

González, G., Sánchez, B.T., \& Márquez, S. (2000).Análisis de los motivos para participar en fútbol y en tenis en la iniciación deportiva. European Journal of Human Movement, (6), 47-66. Retrieved from https: / / dialnet.unirioja.es/servlet/ articulo? codigo $=2278346$

Gould, D., Feltz, D., \&Weiss, M. (1985). Motives for participating in competitive youth swimming. International Journal of Sport Psychology. Retrieved from https:/ / psycnet.apa.org/record/ 1986-21762-001

Gutiérrez-Sanmartín, M., \& Caus-Pertegáz, N.(2006).Análisis de los motivos para la participación en actividades físicas de personas con y sin discapacidad. (Analysis of participation incentives in physical activities among people with and without disabilities.). RICYDE. Revista Internacional de Ciencias Del Deporte, 2(2), 49-64. doi: 10.5232/ricyde2006.00204

Hernández, R., Quiñonez, J.A.,Arenas, J., Urrea,A. M., BarbosaGranados, S., \& Loaiza, H.H.A. (2021). Características Psicológicas en Deportistas con Discapacidad Física (Psychological Characteristicsinathletes with physical disability). Retos, 40(40), 351-358. doi: 10.47197/RETOS.V1I40.83079

Iso-Ahola, S.E., \& Clair, B. S. (2000).Toward a theory of exercise motivation. Quest, 52(2), 131-147. doi: 10.1080/ 00336297.2000 .10491706

Jeong, I., \& Park, S. (2013). Participation motivation and competition anxiety among Korean and non-Korean wheelchair tennis players. Journal of Exercise Rehabilitation, 9(6), 520. doi: $10.12965 /$ jer. 130072

Jimenez,L.E.C., \& Cárdenas,A.I.G. (2020).Validityand confiability of the scale of physical activity for disabled adults PASIPD-C. Retos, 41(41), 162-170. doi: 10.47197/ RETOS.VOI41.77430

K1zar, O., Demir, G.T., \& Genç, H. (2021). Examination of the effect of national and amateur disabled athletes' disabilty types on sports participation motivation. European Journal of Physical Education and Sport Science, 6(12). Retrieved from https: / / www.oapub.org/edu/index.php/ejep/article/view/3655

Lázaro, I.,Villamarín, F. \& \& Limonero, J. (1996). Motivación para participar y auto-eficacia en jóvenes jugadores de baloncesto. IV Congreso Nacional y Andaluz de LaActividad Física y El Deporte. Investigación y Aplicación.

López-Walle, J. M., Balaguer,I., Castillo,I., \&Tristán, J.L. (2011). Climamotivacional percibido, motivación autodeterminaday autoestima en jóvenes deportistas mexicanos. Revista de Psicología Del Deporte, 20(1), 209-222. Retrieved from https: / / www.redalyc.org/articulo.oa:id=235119302015

Marcos, F. M.L., Sánchez, P.A., Sánchez,D.,Amado,D. , \& García, T. (2009). Influencia del clima motivacional creado por el entrenador en el compromiso deportivo en jóvenes jugadores debaloncesto. Cuadernos de Psicología Del Deporte, 9,49-49. Retrieved from https: / / revistas.um.es/cpd/article/view/ 85621

McLoughlin, G.,Weisman Fecske, C., Castaneda,Y., Gwin, C., \& Graber,K. (2017). SportParticipation for EliteAthletesWith Physical Disabilities: Motivations, Barriers, and Facilitators. doi: 10.1123/ apaq.2016-0127

Moreno, J.A., Cervello, E., \& Gonzales,D. (2007). Analizando la motivación en el deporte: un estudio a través de la teoría de la autodeterminación. Colegio Oficial de Psicología, 25(1), 35-51. Retrieved from http: / /www.apuntesdepsicologia.es/ index.php/revista/article/view/113

Muñoz, E. M., Garrote, D., \& Sánchez, C. (2017). La práctica deportiva en personascon discapacidad:motivación personal, inclusión y salud. International Journal of Developmental and Educational Psychology. Revista INFAD de Psicología., 4(1), 145. doi: 10.17060/ijodaep.2017.n1.v4.1037

Pelletier,L. G., Tuson, K. M., Fortier, M. S., Vallerand, R.J., Briére, N.M.,\&Blais, M.R.(1995).Towarda New Measure of Intrinsic Motivation, Extrinsic Motivation, and Amotivation in Sports: The Sport Motivation Scale(SMS). Journal of Sport and Exercise Psychology, 17(1), 35-53.doi: 10.1123/jsep.17.1.35

Silva,A.,Vital,R., \&Túliode Mello,M.(2016).Atuaçãodafisioterapia no esporte paralímpico. Rev Bras Med Esporte, 22(2). doi: 10.1590/1517-869220162202154214

Torralba, M.Á., Braz, M., \& Rubio,M. J.(2014).Lamotivación en el deporte adaptado. Psychology, Society, \& Education, 6(1), 27. doi: $10.25115 /$ psye.v6i1.506

Torralba, M. Á.,Vieira, M. B., \& Rubio, M. J.(2017). Motivos dela práctica deportiva de atletas paralímpicos españoles. Revista de Psicología Del Deporte, 26(1), 49-60. Retrievedfrom https:/ / archives.rpd-online.com/article/view/v26-n1-torralba-vieira-rubio.html

Úbeda-Colomer, J., Molina-Alventosa, P., \& Campos-Granell, J. (2016). Facilitadores y barreras para la prácticafísico-deportiva en alumnado universitario con discapacidad. Educación Física y Deporte, 35(1), 63-96. doi: 10.17533/ udea.efyd.v35n1a03

Urrea, Á. M., \& Guillén, F. (2016). Motivación autodeterminada en deportistas colombianos con discapacidad visual. Katharsis: Revista de Ciencias Sociales, (21), 131-155. Retrieved from https://dialnet.unirioja.es/descarga/articulo/5733139.pdf Vieira,Y.V., Colere, J., \& Souza, D. L. de. (2021). Facilitadores e barreiras para a prática esportiva por parte de atletas com comprometimentos no Brasil (Facilitators and barriers for sports practice by athletes with impairments in Brazil). Retos, 41,812-822. doi: 10.47197/RETOS.V41I0.85497

White, S.A., \&Duda, J.L. (1993). Dimensions of goals and beliefs among adolescent athletes with physical disabilities. Adapted Physical Activity Quarterly, 10(2), 125-136. doi: 10.1123/ apaq.10.2.125 